The scientific culture which pervades the educated classes of Germany is usually ascribed to the efficiency of the Universities ; $I$ believe it would be more correct to put it down to the sound education in scientific matters which the boys receive in the public schools, for it is only the smaller number who proceed to the Universities : the majority go after commercial and industrial pursuits.

University College School, London R. GERSTL

\section{ON DETERMINING THE DEPTH OF THE SEA WITHOUT THE USE OF THE SOUNDING} LINE $^{1}$

THIS is the title of a paper which has been presented to the Royal Society, and Mr. Siemens gave at the meeting of the 24th ult., a description of the instrument which he has designed with this object. He commenced by giving a mathematical statement of the effect of local attraction, to a certain depth, on a body placed at the surface of the earth, assuming it to be of uniform density, spherical in form, and unaffected by centrifugal action. For small values of depth ( $h)$, this attraction is $2 \pi h$, the original formula from which this is adduced is :-

$$
2 \pi h\left(I-\frac{2}{3} \sqrt{\frac{h}{2 R}}\right)
$$

and by substitution of $2 R$ for $h$ in this, Newton's statement of the total attraction $\frac{4}{3} R \pi$ is obtained.

Now, if in place of the solid substance which forms the exterior crust of the earth, whose density may be taken to be the mean density of superficial rock, water, a material of less dancit-y is substituted, it is shown that the total attraction must be diminished, and the measure of this diminution is a measure of the depth of light substance which has been substituted for heavy. If we were in possession of the exact mean density of the earth, of that of the surface-rock, and of sea-water, a scale could be calculated beforehand, to show what depth would agree with a certain diminution of the measured effect of gravitation. Such an approximate calculation was made in designing the instrument, but Mr. Siemens has preferred to compare the readings of the instrument with actual soundings, in order to obtain a scale.

The instrument which is called a bathometer is represented in the accompanying illustration, and consists of the following parts : a weight being a column of mercury affected by variation of gravitation, a counterbalance being springs unaffected by variation of gravitation, ind an arrangement by which the variations in gravitation can be read as depth in units. The column of mercury is maintained in a vertical steel tube having cup-like extensions, the lower portion being closed by a corrugated diaphragm of thin steel plate, and the upper portion containing an aperture for filling the instrument, having a screw stopper. The internal diameter of the tube is reduced at the upper portion, in order that the vertical oscillations of the mercury produced by the motion of a vessel in a sea-way, may be reduced to a minimum, and the instrument is suspended in a universal joint above its centre of gravity, so that it may always hang in a vertical position at sea, and is enclosed in an air-tight casing so that it may not be under the influence of atmospheric changes. The weight of the column of mercury is balanced at the centre of the diaphragm by the elasticity of the steel springs, and the modus operandi of the instrument is evident; as the mercury diminishes in potential through the effects of diminished attraction, the action on the springs diminishes, and these shorten upon themselves.

There are some peculiarities in the mechanical arrangement of the instrument which repay examination. Both ends being open to the air, its indications are not By C. William Siemens, D.C.L., F.R.S. affected by variations of atmospheric pressure. With regard to temperature, the instrument is parathermal.

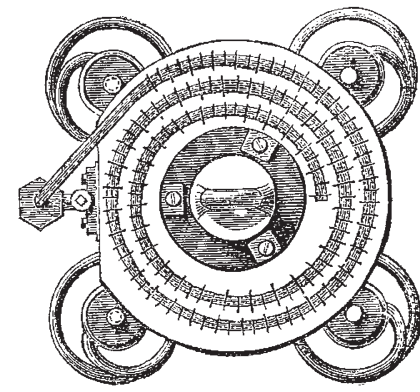

Its peculiar form has been the result of scientific inquiry. It was first discovered by experiment that well-tempered

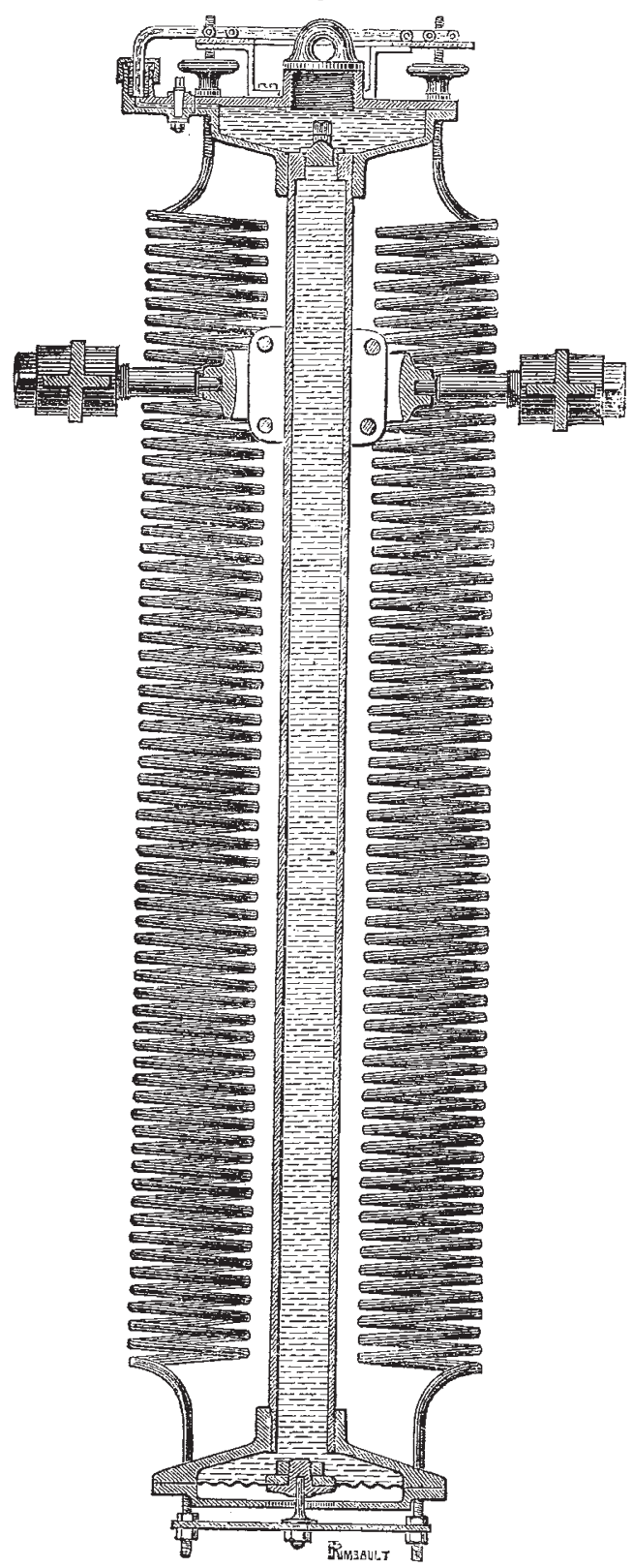

steel springs diminished in potential with rise of temperature in a constant ratio, it was therefore necessary that 
the potential of the mercury should diminish in the same ratio. Mercury contained in a tube of uniform section would always have the same potential, for as it expanded and lengthened by heat, its specific density would diminish, and the product of density into height would remain constant. If contained in a tube of infinitely small diameter, compared with the diameter of the cup-like extensions, the height would remain constant, whilst the potential would diminish in the ratio of the expansion of mercury, but this is different from the ratio of the diminution of potential of the springs, and in order that these ratios may be accordant, or in other words that the equilibrium of the whole system may be the same at all temperatures, the peculiar form has been employed represented in the illustration, which is between the two forms already referred to.

The amount of the variation of gravitation with variation of depth is exceedingly small, and requires some method for indicating it. The method employed in the results hitherto tabulated and presented with the paper is by means of electrical contact, which is established whenever a sounding is to be taken, between the centre of the steel diaphragm and the end of a micrometer screw, which is at other times insulated from the body of the instrument. The screw is of such pitch, and the circular plate which turns with it has divisions so proportioned, that each division represents a depth of one fathom.

The readings of the instrument have been compared with soundings taken by means of Sir Wm. Thomson's steel wire sounding apparatus, and the accordance between the two is very satisfactory, especially as the bathometer, from the very nature of its action, gives a mean of the surrounding depths, whilst the soundingline gives the actual depth below the ship. The reading of the instrument is also effected by means of a spiral glass tube, connecting by means of liquid, less dense than mercury, with the mercury in the upper cup ; this arrangement has lately been tried and found to work successfully. The instrument is also available for the measurement of height ; in mountain ascents, however, the elevated land will influence its readings, and allowance would have to be made for the effect of this local attraction.

The chief disturbing element in the use of this instrument is the effect of latitude, which will have to be ascertained approximately before its readings can be accepted as true indications of the depth. The difference between the total attraction of the earth at the pole and on the equator amounts to $\frac{1}{180}$ of its effect at the equator, the rate of increase in travelling from the equator to the pole being as the square of the sine of the latitude. The amount of this variation is easily calculable in fathoms of depth, to be tabulated for use with the instrument.

The principal value of the bathometer would be to serve the mariner as an additional means of determining his position when he was debarred from taking astronomical observation on account of the state of the weather. If the contour of the ocean bed were laid down on charts more perfectly than it is at present, and if these were in the hands of the mariner, he would be able to tell from his bathometer what was the depth of ocean below him, and whether that depth was increasing or decreasing in pursuing his course; he could also observe the rate of increase or decrease of depth, and in consulting his chart he would be enabled to determine his actual position with considerable accuracy, and thus be forewarned of the approach of danger.

PHOTOGRAPHY OF THE RED AND ULTRARED END OF THE SPECTRUM

N 1874 Dr. Vogel communicated to the scientific public that he had been able to plotograph the least refrangible rays of the solar spectrum by using what are known as the Uranium Dry Plates prepared by the
Uranium Dry Plate Company. At the same time he experimented with other bromide plates, using dyes to give them distinctive tints. He then enunciated that the sensitiveness of the plates for the hitherto unphotographed portion of the spectrum was due to the colours employed, and apparently all his efforts have been diverted in elaborating this idea. Last summer I commenced a series of similar experiments to see whether the discovery could be made of practical use in photography; and have arrived at the conclusion, that the colouring matter gives this extended sensitiveness owing to the compound of silver formed and not to the colour itself, in fact, that the tint given to the film necessitates a very prolonged exposure. The additions of resins, nitro-glucose, and other similar compounds containing carbon to the bromized and bromoiodized collodion soon convinced me that it was the organic salts of silver to which we must look for sensitiveness in the yellow red and ultra-red rays of the spectrum. Nearly every resinous body seems to prolong the photographic spectrum towards the ultra-red, and one or two in particular, also when the film is least colourless when viewed by transmitted light, that then it is probably in the most impressionable condition. Another point which is worthy of notice is, that a film dried from moisture, taking, in fact, the form of a dry plate, is always most readily acted upon by the ultra-red end of the spectrum. Probably this is due to the absorptive qualities possessed by the silver nitrate solution, and not really from an increased sensitiveness of the compound salts when dry.

If ordinary pyroxylin be employed for the collodion it is generally less suitable than if it contain a certain quantity of nitro-glucose, or other similar body ; and it frequently happens if this be absent entirely that the photographic spectrum will stop short near $b$.

Taking a collodion made with pyroxylorin prepared at high temperatures and using the ordinary solvents (in the alcohol of which suitable resin and bromides are dis. solved), it - will be found in general that when the silver salts formed through them by the sensitizing bath, or by emulsifying them by direct addition of silver nitrate to the collodion, are presented to the action of the spectrum, the whole of it will be impressed with a developable image. With three prisms of $60^{\circ}$ and one of $45^{\circ}$ twenty minutes is sufficient exposure to give when the slit is nearly closed, a lens of four feet focus being used as the objective.

When this is overcome it will be possible (and I hope shortly to do so) to present a complete photographic map of those lines which lie beyond $A$ to a distance at least equal to $D-A$, a point beyond which $I$ have not as yet been able to obtain an image. The great difficulty to be encountered is that of finding a sharp focus for the different points of the invisible spectrum, the change in length from one point to another being very rapid.

Uranium and iron salts have also furnished me with spectra which are well worthy of notice. With the latter salt more especially the action of the beat-rays is very decided, though at present it seems to me that the exposure must be very prolonged.

I propose at a later date to give details of all the most interesting of these experiments sufficient to enable anyone to repeat them who may desire to do so.

$$
\text { W. DE W. ABNEY }
$$

P.S.- It may be as well to state that the best results with resin plates have been obtained when a modification of alkaline development has been adopted.

\section{RAOUL PICTET'S SULPHUROUS ACID ICE- MACHINE}

THE countries between the 4oth degrees of $\mathrm{N}$. and $\mathrm{S}$. latitude have in general too temperate winters to admit of natural ice being obtained in any quantity; and yet these are the countries in which it is most required. 